\title{
Acquired Resistance to Borrelia burgdorferi Infection in the Rabbit Comparison Between Outer Surface Protein A Vaccine- and Infection-derived Immunity
}

\author{
Denise M. Foley, ${ }^{\star}$ Yi-Ping Wang, ${ }^{\star}$ Xiao-Yang Wu, ${ }^{*}$ David R. Blanco, ${ }^{*}$ Michael A. Lovett, ${ }^{\star \ddagger}$ and James N. Miller \\ $*$ Department of Microbiology and Immunology, and ${ }^{\ddagger}$ Division of Infectious Diseases, Department of Medicine, University of California, \\ Los Angeles, School of Medicine, Los Angeles, California 90024
}

\begin{abstract}
Intradermal inoculation of the rabbit with Borrelia burgdorferi, sensu lato, results in the consistent development of erythema migrans (EM), dermal infection, and visceral dissemination of the spirochete. Within 5 mo, EM as well as dermal and visceral infection are cleared and the animals exhibit immunity to reinfection. This study compares infection-derived immunity with acquired resistance resulting from the administration of a lipidated recombinant outer surface protein A (OspA) vaccine presently undergoing human trial. 4 of 11 OspA vaccinated rabbits, challenged intradermally at each of 10 sites with $10^{5}$ low passage $B$. burgdorferi, developed EM as well as dermal and disseminated infection. After identical challenge, 2 of the 11 infectionimmune rabbits developed a dermal infection, but not EM or disseminated infection. Further, ELISA anti-OspA titers did not correlate with the status of immunity for either OspA vaccinated or infection-immune rabbits. Prechallenge ELISA anti-OspA titers were relatively low in the infection-immune group. This study demonstrates that a state of partial immunity to experimental Lyme disease may result that could potentially mask infection. Further, our data strongly suggest that immunogen(s) other than OspA is/are responsible for stimulating acquired resistance in the infection-immune rabbit. (J. Clin. Invest. 1997. 99:2030-2035.) Key words: Lyme borreliosis $\bullet$ protective immunity $\bullet$ rabbit model $\bullet$ spirochete $\cdot$ vaccinogens
\end{abstract}

\section{Introduction}

Lyme disease, the most common vector-borne disease in the United States (1), is caused by the spirochete Borrelia burgdorferi, sensu lato (2). The disease is transmitted to humans most often by the bite of an infected Ixodes tick and is characterized by the initial appearance of distinctive erythema migrans $(E M)^{1}$ skin lesions in $60-80 \%$ of patients $(3,4)$. Key

Address correspondence to James N. Miller, Department of Microbiology and Immunology, UCLA School of Medicine, 10833 Le Conte Avenue, Los Angeles, CA 90024-1747. Phone: 310-825-1979; FAX: 310-206-3865.

Received for publication 14 November 1996 and accepted in revised form 5 February 1997.

1. Abbreviations used in this paper: EM, erythema migrans; OspA, outer surface protein A.

J. Clin. Invest.

(C) The American Society for Clinical Investigation, Inc. 0021-9738/97/04/2030/06 \$2.00

Volume 99, Number 8, April 1997, 2030-2035 early and late clinical manifestations reflecting dissemination to visceral targets include lymphadenopathy, arthritis, neurological manifestations, and carditis (3-14). Periods of chronicity and recurrent attacks in the untreated patient often occur $(11,14)$. The development and evaluation of an efficacious vaccine against this complex disease and its etiologic agent mandate a thorough understanding of spirochetal virulence and pathogenesis. Although characterization of B. burgdorferi surface molecules is far from complete, attempts have been made to develop effective vaccinogens using membrane lipoproteins (15-24). The major focus has been on the most abundant of these lipoproteins, designated outer surface protein A (OspA), which has been shown to be predominantly located in the periplasmic space (25). OspA has been successful in inducing a high degree of protective immunity in mice and dogs against needle and/or heterologous tick challenge (15-18, 20 24). It has been shown that after tick challenge, the antibodies generated by these vaccinated animals are sufficient to eliminate the spirochetes in the tick vector before they can infect the host $(21,22)$.

In previous studies, we have shown for the first time that EM, as well as persistent skin infection and visceral dissemination, can be induced in the rabbit after intradermal injection of B. burgdorferi (26). Within $5 \mathrm{mo}$, the infection clears and the animals exhibit a high degree of immunity to reinfection (26). Thus, the rabbit provides a unique model in which protective immunity to $B$. burgdorferi can be assessed whether acquired by infection or by vaccination with individual or combined $B$. burgdorferi molecules.

In an effort to determine whether molecules other than the recombinant OspA presently being evaluated in human trials may be efficacious as protective immunogens, we conducted a study in the rabbit in which we compared OspA and infectionderived immunity. We show that intradermal challenge of infection-immune rabbits with $10^{6}$ B. burgdorferi, strain B31, sensu stricto, results in a greater degree of homologous protection compared with that obtained in the OspA vaccinated rabbits. The status of immunity among both the OspA vaccinated and infection-immune groups did not correlate with antiOspA antibodies as measured by ELISA. Further, completely resistant infection-immune rabbits exhibited significantly lower prechallenge titers than susceptible OspA vaccinated rabbits at the same timepoint. These data support the concept that efficacious vaccines can be developed that circumvent the limitations associated with OspA immunization.

\section{Methods}

Experimental animals. Adult male New Zealand white rabbits 6-9 mo of age (Irish Farms, Norco, CA) were housed individually in a temperature controlled environment ranging from $18-21^{\circ} \mathrm{C}$. Before each intradermal inoculation and challenge with $B$. burgdorferi, the backs of the rabbits were clipped closely with an electric clipper fitted 
with a size 40 blade to expose the skin (Oster Professional Products, McMinnville, TN).

Immunization of rabbits with OspA. Rabbits were vaccinated with a recombinant OspA lipoprotein adsorbed onto aluminum hydroxide (alum) and with alum alone kindly supplied by SmithKline Beecham Biologicals (Rixensart, Belgium). The vaccinogen and alum preparations were diluted to appropriate concentration with PBS buffer containing $5 \mathrm{mM} \mathrm{K}_{2} \mathrm{HPO}_{4}, 5 \mathrm{mM} \mathrm{NaH}_{2} \mathrm{PO}_{4}$, and $150 \mathrm{mM} \mathrm{NaCl}$ adjusted to $\mathrm{pH}$ 6.7. Rabbits received three intramuscular injections administered at 2-wk intervals; each injection contained $10 \mu \mathrm{g}$ of OspA vaccine in $1.0 \mathrm{ml}$ of PBS diluent, $\mathrm{pH}$ 6.7. As sham controls, rabbits were inoculated as described with a total of three doses of alum contained in $1.0 \mathrm{ml}$ of diluent per dose.

Production of infection-derived immune rabbits. On the basis of previous studies in which a high degree of infection-derived immunity to challenge was established (26), rabbits were infected intradermally with $6 \times 10^{7}$ virulent $B$. burgdorferi, strain B31, sensu stricto, passage 4. 7 mo after infection, at a time when EM and dermal infection could not be detected by culture of skin biopsies and, on the basis of previously described studies, visceral dissemination was no longer demonstrable (26), the rabbits were challenged as described below.

Preparation of the challenge inoculum. Virulent B. burgdorferi, strain B31, sensu stricto, was isolated from infected rabbit tissue, grown in BSK II medium (27) to maximum density, and then passaged twice more in fresh medium. After the final passage (passage 2 ), the organisms were centrifuged, washed three times in heat-inactivated normal rabbit serum diluted 1:1 in PBS, pH 7.2 (NRS-PBS), allowed to sit at room temperature for 15 minutes after each centrifugation and resuspension, and then adjusted with NRS-PBS to the concentration required for challenge after the final wash (26).

Challenge with B. burgdorferi. $4 \mathrm{wk}$ after the final booster, the immunized, sham control, naive control, and infection-derived immune rabbits were challenged intradermally at 10 sites on the clipped back. Each site received $10^{5} \mathrm{~B}$. burgdorferi, strain B31, sensu stricto, passage 2 contained in $0.1 \mathrm{ml}$ of modified BSK II prepared as described above. The animals were examined daily for the presence of EM lesions. At defined time points described below, skin biopsies for culture were obtained from both representative EM lesions and from representative dermal sites of those animals in which EM lesions did not appear. Similarly, popliteal lymph nodes, joint tissue, and spinal cord were removed from representative rabbits in each group and cultured as described below. For future immunopathogenesis studies, skin biopsies were obtained from 17 and 16 random sites on four rabbits in each of the sham and naive control groups, respectively, before the expected time of EM appearance. As a result, these sites could not be included in the observations relating to EM formation. The presence or absence of EM at an additional two sites on two sham vaccinated rabbits could not be determined due to heavy fur growth at the sites.

Skin biopsy procedures. Skin biopsies were carried out on random sites on each rabbit at the time of EM development in the control animals (usually $7 \mathrm{~d}$ ) and at 19-22 d postchallenge. Rabbits were anesthetized by intramuscular injection with $45 \mathrm{mg}$ Ketaset (Fort Dodge Laboratories, Fort Dodge, IA) and $8.8 \mathrm{mg}$ Xylazine (Lloyd Laboratories, Shenandoah, IA) per kilogram body weight. Clipped backs were disinfected with $70 \%$ ethanol and $3 \%$ hydrogen peroxide. Two 4-5-mm punch biopsies adjacent to the inoculation site (Baker and Cummings, Lakewood, NJ) were taken from each rabbit. Each biopsied specimen was immediately placed on ice in a sterile Petri dish and then processed within $1 \mathrm{~h}$ for culture and future studies.

Tissue removal for dissemination studies. At 19-22-d postchallenge, rabbits were anesthetized as described above and exsanguinated from the central artery. The popliteal lymph nodes, spinal cord, and joint tissue surrounding the patella were aseptically removed immediately upon killing, placed on ice in a sterile Petri dish, and then processed for culture and future studies within $2 \mathrm{~h}$. The spinal cord was exposed by removal of the dorsal portion of the vertebrae with sterile instruments. After exposure, the cord was raised with a sterile spatula, severed with a sterile scalpel, and a portion removed. For joint removal, the skin over the surface of the hind legs of the rabbit was clipped with an electric clipper, sterilized with $70 \%$ ethanol, and removed. The tendons above the patella were severed with a sterile scalpel and the joint tissue was then removed with a sterile forceps and sterile scalpel.

Culture of tissues from immunized and control rabbits. Representative portions of each tissue were divided into $\sim 5$-mm sections, minced, and then placed into $5 \mathrm{ml}$ of BSK II medium containing 100 $\mu \mathrm{g} / \mathrm{ml}$ phosphomycin and $50 \mu \mathrm{g} / \mathrm{ml}$ rifampin (28). Cultures were incubated at $34^{\circ} \mathrm{C}$ for a period of $30 \mathrm{~d}$, and the presence or absence of $B$. burgdorferi was determined by darkfield microscopy. Cultures were considered negative when no spirochetes were observed during the 30-d observation period. $100 \mu \mathrm{l}$ of blood was cultured and examined in a similar fashion.

Sera for ELISA. Blood was obtained aseptically from the central artery of the control and experimental rabbits before vaccination (basals), before challenge, and at postchallenge time points. The sera were separated by centrifugation, aliquoted, and stored individually at $-70^{\circ} \mathrm{C}$ until ready for use.

ELISA. The ELISA was carried out as previously described with minor modifications (26). Flat bottomed, 96-well immunoassay plates (Immulon 4; Dynatech Laboratories, Inc., Chantilly, VA) were coated overnight at room temperature in a humidified chamber with $100 \mu \mathrm{l}$ of $1 \mu \mathrm{g} / \mathrm{ml}$ recombinant OspA antigen kindly supplied by SmithKline Beecham Biologicals. The plates were washed twice with wash buffer ( $0.5 \%$ Tween 20 in PBS) and blocked for $1 \mathrm{~h}$ with Blotto (5\% dry nonfat milk in PBS). After two washes with wash buffer, $100 \mu$ l of twofold dilutions of rabbit serum in PBS ranging from 1:500 to 1:320,000 were added in duplicate to the wells and incubated at room temperature for $2 \mathrm{~h}$. Plates were washed four times, incubated with goat antirabbit immunoglobulin conjugated to horseradish peroxidase, and diluted to 1:5,000 or 1:2,000, respectively (Amersham Corp., Arlington Heights, IL). The plates were then washed four times and $100 \mu \mathrm{l}$ of 2,2'-azinobis (3-ethyl benzthiazoline sulfonic acid) peroxidase substrate system (Kirkegaard \& Perry Laboratories, Inc., Gaithersburg, MD) was added to each well. After $30 \mathrm{~min}$, the OD of each well was measured at $405 \mathrm{~nm}$ on an automated ELISA plate reader (Titertek Multiscan MCC/340; Flow Laboratories, Baar, Switzerland). Serum from an OspA immunized rabbit was chosen as a positive standard to measure interassay variability. This serum was run in serial dilution on each plate, and the mean and standard deviation of each dilution determined; the standard deviation was found to be $\leq 0.03$ at the tested dilutions.

Statistical analyses. Proportions were compared by the chi square method. Mean log anti-OspA ELISA titers were compared by the $t$ test. Statistical significance was determined by $P<0.05$.

\section{Results}

Protection of rabbits conferred by $O \operatorname{sp} A$ vaccination. As shown in Table I, OspA vaccination resulted in statistically significant but incomplete protection after challenge with $B$. burgdorferi, strain B31, sensu stricto, compared with the challenged naive and sham-inoculated control rabbits (chi square value $=19.75$; degree of freedom [d.f.] $=2 ; P=0.0001)$. 4 of 11 rabbits vaccinated with the recombinant OspA lipoprotein vaccine and challenged Id at each of 10 sites per rabbit with $10^{5} \mathrm{~B}$. burgdorferi, strain B31, passage 2, developed culture-positive EM lesions at one to four sites. In contrast, 12 of 12 sham-vaccinated and 12 of 12 naive control rabbits developed EM lesions at 98 of 101 and 103 of 104 challenged sites, respectively. No differences in the time of appearance, duration, or diameter of the EM lesions were observed between the OspA-vaccinated, sham-vaccinated, and naive rabbits. Among the 11 OspA-vaccinated rabbits, dermal infection was demonstrable only 
Table I. Comparative Degree of Protection Between OspA Vaccinated and Infection-immune Rabbits After Challenge with B. burgdorferi, Strain B31, Sensu Stricto

\begin{tabular}{|c|c|c|c|c|c|}
\hline \multirow[b]{2}{*}{ Rabbit number } & \multirow{2}{*}{$\begin{array}{l}\text { Number of EM } \\
\text { lesions*/total sites } \\
\text { observed }\end{array}$} & \multicolumn{4}{|c|}{$\begin{array}{l}\text { Number of positive cultures/ } \\
\text { total sites cultured }\end{array}$} \\
\hline & & Skin & $\begin{array}{c}\text { Popliteal } \\
\text { lymph nodes }\end{array}$ & Joint tissue & Spinal cord \\
\hline \multicolumn{6}{|c|}{ OspA vaccinated group } \\
\hline A2 & $1 / 10$ & $8 / 8$ & $2 / 2$ & $2 / 2$ & $0 / 1$ \\
\hline $\mathrm{A} 3$ & $2 / 10$ & $7 / 8$ & $2 / 2$ & $2 / 2$ & $0 / 1$ \\
\hline A8 & $4 / 10$ & $8 / 9$ & $2 / 2$ & $2 / 2$ & $1 / 1$ \\
\hline A7 & $1 / 10$ & $7 / 9$ & $1 / 2$ & $2 / 2$ & $0 / 1$ \\
\hline A1 & $0 / 10$ & $0 / 9$ & $0 / 2$ & $0 / 2$ & $0 / 1$ \\
\hline A9 & $0 / 10$ & $0 / 5$ & $\mathrm{NC}$ & $\mathrm{NC}$ & $\mathrm{NC}$ \\
\hline A10 & $0 / 10$ & $0 / 5$ & $\mathrm{NC}$ & $\mathrm{NC}$ & $\mathrm{NC}$ \\
\hline A4 & $0 / 10$ & $0 / 9$ & $0 / 2$ & $0 / 2$ & $0 / 1$ \\
\hline A13 & $0 / 10$ & $0 / 12$ & $\mathrm{NC}$ & $\mathrm{NC}$ & $\mathrm{NC}$ \\
\hline A11 & $0 / 10$ & $0 / 4$ & $\mathrm{NC}$ & $\mathrm{NC}$ & $\mathrm{NC}$ \\
\hline A5 & $0 / 10$ & $0 / 9$ & $0 / 2$ & $0 / 2$ & $0 / 1$ \\
\hline \multicolumn{6}{|c|}{ Infection-immune group } \\
\hline DC1 & $0 / 10$ & $4 / 11$ & $0 / 2$ & $0 / 2$ & $0 / 1$ \\
\hline $\mathrm{DC} 2$ & $0 / 10$ & $1 / 11$ & $0 / 2$ & $0 / 2$ & $0 / 1$ \\
\hline DC5 & $0 / 10$ & $0 / 9$ & $0 / 2$ & $0 / 2$ & $0 / 1$ \\
\hline DC11 & $0 / 10$ & $0 / 9$ & $0 / 2$ & $0 / 2$ & $0 / 1$ \\
\hline DC3 & $0 / 10$ & $0 / 9$ & $\mathrm{NC}$ & $\mathrm{NC}$ & $\mathrm{NC}$ \\
\hline DC13 & $0 / 10$ & $0 / 9$ & $0 / 2$ & $0 / 2$ & $0 / 1$ \\
\hline DC22 & $0 / 10$ & $0 / 4$ & $\mathrm{NC}$ & $\mathrm{NC}$ & $\mathrm{NC}$ \\
\hline DC26 & $0 / 10$ & $0 / 4$ & $\mathrm{NC}$ & $\mathrm{NC}$ & $\mathrm{NC}$ \\
\hline $\mathrm{DC} 12$ & $0 / 10$ & $0 / 9$ & $0 / 2$ & $0 / 2$ & $0 / 1$ \\
\hline DC4 & $0 / 10$ & $0 / 9$ & $0 / 2$ & $0 / 2$ & $0 / 1$ \\
\hline DC15 & $0 / 10$ & $0 / 4$ & $\mathrm{NC}$ & $\mathrm{NC}$ & $\mathrm{NC}$ \\
\hline \multicolumn{6}{|c|}{ Sham vaccinated group } \\
\hline B2-B13 & 98/101 & $82 / 82$ & $14 / 14$ & $12 / 13$ & $3 / 7$ \\
\hline \multicolumn{6}{|c|}{ Naive control group } \\
\hline $\mathrm{C} 1, \mathrm{C} 3-\mathrm{C} 13$ & $103 / 104$ & $82 / 85$ & $9 / 14$ & $13 / 14$ & $4 / 7$ \\
\hline
\end{tabular}

NC, not cultured. *Cultured EM lesions were always positive.

among the four animals that developed EM lesions. Further, among the seven OspA-vaccinated animals assayed for evidence of visceral dissemination, only the four rabbits that developed EM lesions showed culture-positive popliteal lymph nodes, joint tissue, and/or spinal cord at 19-22 d postchallenge. Again, this is in contrast to the sham immunized and naive control rabbits, each of which developed culture-positive skin and viscera. It should be noted that one of the four susceptible OspA-vaccinated rabbits (A2) received only two vaccine doses.

Protection of rabbits conferred by infection. A statistically significant degree of acquired resistance was also demonstrable among the challenged infection-immune rabbits compared with the challenged naive control animals (chi square value $=$ 17; d.f. $=2 ; P=0.0001$ ). As shown in Table I, only 2 of the 11 infection-derived immune rabbits showed evidence of infection; each of the remaining 9 rabbits was completely immune to EM and dermal infection, while the 7 animals in this group that were tested showed complete immunity to visceral dissemination. Even among the two animals that failed to exhibit a complete immunity, the organisms did not generate an EM response, produced dermal infection at only 5 of 22 cultured sites, and remained localized to the skin.

Comparative degree of protection between OspA vaccinated and infection-immune rabbits. A higher degree of resistance against B. burgdorferi challenge was observed among the infection-immune rabbits ( 9 of $11=81.8 \%$ ) compared with OspA vaccinated animals ( 7 of $11=63.6 \%$ ). However, this difference was not statistically significant $(P>0.05)$. Nonetheless, it should be noted that while 4 of 11 OspA vaccinated rabbits developed both EM and dissemination, none of the 11 challenged infection-immune rabbits exhibited these clinical manifestations (chi square value $=4.9$; d.f. $=1 ; P=0.03$ ).

Relationship between status of immunity and ELISA antiOspA antibody titers. No significant differences in prechallenge ELISA anti-OspA titers were observed between the susceptible and immune OspA vaccinated rabbits $(P>0.05)$. Prechallenge titers of 1:320,000 were observed in two of the four susceptible and four of the seven immune rabbits (Table II).

There was also no correlation found between the status of immunity of the infection-immune rabbits and their prechallenge ELISA anti-OspA titers $(P>0.05)$. The two animals

Table II. Relationship Between the Status of Immunity and ELISA Anti-OspA Antibody Titers Among OspA Vaccinated and Infection-immune Rabbits Challenged with B. burgdorferi, Strain 31, Sensu Stricto

\begin{tabular}{|c|c|c|c|c|c|c|}
\hline \multirow{3}{*}{ Group } & \multicolumn{3}{|c|}{$\begin{array}{l}\text { ELISA anti-OspA titers } \\
\text { (reciprocal dilution) }\end{array}$} & \multicolumn{3}{|c|}{$\begin{array}{l}\text { Infected tissue } \\
\text { after challenge* }\end{array}$} \\
\hline & \multirow[t]{2}{*}{ Prechallenge } & \multicolumn{2}{|c|}{ Postchallenge } & \multirow[t]{2}{*}{ EM } & \multirow[t]{2}{*}{ Skin } & \multirow[t]{2}{*}{ Viscera } \\
\hline & & $7 d$ & $3-4 w k$ & & & \\
\hline \multirow{11}{*}{$\begin{array}{l}\text { OspA } \\
\text { vaccinated } \\
\text { rabbits }\end{array}$} & 40,000 & 40,000 & 80,000 & + & + & + \\
\hline & 80,000 & 80,000 & 40,000 & + & + & + \\
\hline & 320,000 & 40,000 & 80,000 & + & + & + \\
\hline & 320,000 & 160,000 & 40,000 & + & + & + \\
\hline & 160,000 & 160,000 & 160,000 & - & - & - \\
\hline & 160,000 & 80,000 & 80,000 & - & - & $\mathrm{NC}$ \\
\hline & 160,000 & 80,000 & 40,000 & - & - & $\mathrm{NC}$ \\
\hline & 320,000 & 80,000 & 160,000 & - & - & - \\
\hline & 320,000 & 80,000 & 40,000 & - & - & $\mathrm{NC}$ \\
\hline & 320,000 & 80,000 & 80,000 & - & - & $\mathrm{NC}$ \\
\hline & 320,000 & 160,000 & 160,000 & - & - & - \\
\hline \multirow{11}{*}{$\begin{array}{l}\text { Infection- } \\
\text { immune } \\
\text { rabbits }\end{array}$} & 5,000 & 20,000 & 40,000 & - & + & - \\
\hline & 10,000 & 5,000 & 40,000 & - & + & - \\
\hline & 1,000 & 40,000 & 40,000 & - & - & - \\
\hline & 2,000 & 20,000 & 80,000 & - & - & - \\
\hline & 2,000 & 10,000 & 80,000 & - & - & $\mathrm{NC}$ \\
\hline & 5,000 & 10,000 & 40,000 & - & - & - \\
\hline & 10,000 & 20,000 & 20,000 & - & - & $\mathrm{NC}$ \\
\hline & 10,000 & 20,000 & 20,000 & - & - & $\mathrm{NC}$ \\
\hline & 10,000 & 10,000 & 40,000 & - & - & - \\
\hline & 10,000 & 5,000 & 80,000 & - & - & - \\
\hline & 20,000 & ND & 40,000 & - & - & $\mathrm{NC}$ \\
\hline
\end{tabular}

Basal, prechallenge, and postchallenge sera from 13 rabbits each in the sham-vaccinated and naive control groups, as well as the basal sera from the 11 OspA vaccinated and 11 infection-immune rabbits, exhibited titers of $\leq 1: 500$. ${ }^{*}-$, negative culture; + , positive culture; $\mathrm{NC}$, not cultured; ND, not done. 
susceptible to dermal infection upon challenge exhibited titers of 1:5,000 and 1:10,000, whereas among the nine rabbits immune to EM, dermal infection, and/or visceral dissemination, only one showed a higher titer $(1: 20,000)$; the remaining immune animals exhibited titers ranging from $1: 1,000$ to $1: 10,000$ (Table II).

Similarly, there was no correlation between immune status and ELISA anti-OspA titers observed among both the OspA vaccinated and infection-immune animals at $7 \mathrm{~d}$ and 3-4 wk postchallenge $(P>0.05$; Table II).

\section{Discussion}

We recently described a rabbit model of Lyme disease with two novel features (26). First, intradermal injection of B. burgdorferi consistently induced lesions with the appearance and histological features of erythema migrans. The rabbit is the only animal model besides the rhesus monkey (29) that produces EM, and the only model in which EM consistently appears. Second, within 5 mo of infection, rabbits had cleared EM as well as dermal and visceral infection and exhibited immunity to reinfection (26). In this report, we have employed each of these key features of the rabbit model in comparing infection-derived immunity with immunity acquired by immunization with OspA.

Our findings represent the first extensive assessment of EM with regard to protection conferred by an OspA vaccine in an animal model. As in many studies with rodents (15-18, 21, 22), OspA immunization of rabbits conferred significant resistance against $B$. burgdorferi infection. The fact that the protection was incomplete is of considerable interest. It should be noted that early studies with OspA immunization of mice demonstrated that complete protection of mice was evident when $10^{4}$ or fewer spirochetes were used in intradermal or intraperitoneal challenge. However, challenge with larger numbers of B. burgdorferi resulted in incomplete or no protection (17). Subsequent studies have used challenge doses of $10^{4}$ or fewer $(16,30)$. In our previous characterization of infectionderived immunity, rabbits challenged with as many as $8 \times 10^{7}$ virulent $B$. burgdorferi demonstrated solid immunity. In this study, we challenged rabbits with considerably fewer organisms, $10^{5}$ at each of 10 sites for a total of $10^{6} \mathrm{~B}$. burgdorferi per rabbit. When comparing the high dose of challenge that can be administered to previously infected animals that subsequently demonstrate complete protection with our findings of partial protection conferred by OspA immunization, the contrast is of particular interest. While each of the four OspA immunized rabbits susceptible to infection exhibited EM, it is noteworthy that EM occurred at relatively few (1-4) of the 10 intradermal inoculation sites. While the appearance of EM at the inoculated sites was variable, dermal infection and dissemination were evident in each of these four animals. The two infected rabbits in the infection-immune group developed dermal infection without EM or visceral infection. Taken together, these observations indicate that states of partial immunity may be acquired in the rabbit; in the partially immune animal, protection against EM could be accompanied by chronic skin and/or visceral infection with $B$. burgdorferi.

Lyme disease in humans is a chronic infection which, after months to years, may lead to disease manifestations such as arthritis, neurological illness, and carditis (3-14). As a result, the potential consequences of vaccination resulting in partial im- munity, namely, establishment of infection without the development of EM, must be considered. In this study, we report that two infection-derived immune rabbits developed dermal infection upon challenge despite failure to develop EM lesions and visceral dissemination (Table I). A vaccine that permits the survival of organisms in tissues without producing EM after exposure allows the establishment of chronicity in the absence of the characteristic skin lesion that prompts patients to seek treatment. This potential "masking" could result in the later development of debilitating disease. Further, these observations, together with our finding that complete infectionderived immunity can wane after 7 mo with only dermal infection upon challenge, emphasize the necessity for evaluating the need for booster doses. Of further relevance are the potential consequences of vaccination that may lead to seronegative infection. Luft et al. have described how unsuccessful treatment of EM with azithromycin led to recurrent infection marked by seronegativity, while those patients in whom azithromycin or amoxicillin prevented relapse were more likely to become seropositive (31). These data also support the possibility that a state of partial immunity generated by vaccination may "mask" infection that develops in the absence of EM and seropositivity.

We cannot definitively address why OspA vaccination did not confer complete immunity. As considered above, we suspect that greater protection would have been apparent had lesser numbers of B. burgdorferi been used in the challenge inoculum. There was no statistical difference between the ELISA anti-OspA titers of the immune and susceptible rabbits. However, it is possible that antibodies to OspA epitopes most closely linked with borreliacidal activity (32) did exhibit significant variance among the animals and may relate causally to the varying degrees of protection observed. Unlike inbred mice used in the rodent challenge experiments (15-18, 21-24), rabbits are outbred animals and would be expected to respond to antigens in a more variable manner.

Our findings regarding infection-derived immunity confirm and extend our initial observations (26), where complete protection against challenge with $8 \times 10^{7}$ low passage $B$. burgdorferi, strain B31 was observed 5 mo after initial infection. In this study, challenge with $10^{6}$ low passage $B$. burgdorferi, strain B31 was conducted 7 mo after infection. Highly significant protection resulted but, because skin infection in the absence of EM and dissemination resulted in 2 of the 11 rabbits, infection-derived immunity may have waned after 7 mo. There was no statistically significant difference between the numbers of fully immune and susceptible rabbits in the infection-immune and OspA vaccinated groups. However, the observation that all the susceptible OspA rabbits developed EM and disseminated infection while the infection-immune group developed only a dermal infection is significant. This may perhaps indicate a greater degree of protective immunity in the infection immune rabbits.

While the relative degree of protection acquired by OspA vaccination compared with that acquired by previous infection may be arguable, the difference in prechallenge anti-OspA titers between the groups was striking. ELISA anti-OspA titers ranged from $1: 1,000$ to $1: 20,000$ in the infection-immune rabbits compared with 1:160,000 to 1:320,000 in the immune OspA vaccinated rabbits (Table II). These findings are consistent with the possibility that acquired resistance may be generated by molecules other than OspA. We have recently identified sev- 
eral virulent associated outer membrane proteins that might be targets of protective immunity (33). Other investigators have obtained data that are in agreement with this observation (34, 35). In the study conducted by Barthold and Bockenstedt (36), mice were passively protected with mouse sera generated from mice injected with varying doses of inoculum. They concluded that the protection generated appeared to be unrelated to antibody to OspA. In the study conducted by Aydintug et al. (34), they observed that sera obtained from monkeys infected by tick feeding demonstrated antibody-dependent complementmediated killing despite the lack of anti-OspA and -OspB antibodies in this serum.

While OspA has been shown to have considerable efficacy as a vaccinogen by several investigators (15-24), there has been a growing appreciation of the potential limitations in its use in this regard. Recently, evidence has accumulated that OspA is not expressed during vertebrate infection (37), and OspA vaccinated inbred mice have been shown to be susceptible to infection derived from skin implants taken from infected syngeneic animals (38). The antigenic heterogeneity of OspA among strains (39-42) is well known, and the potential for significant numbers of OspA escape mutants after vaccination and challenge have been described (40), along with the failure to provide cross protection by needle inoculation against strains with OspA genotypes different from the vaccinogen $(23,30)$. In addition, $B$. burgdorferi may persist in animals with relatively high antibody titers $(34,42$, and this study), and a decline of anti-OspA antibody titers occurs with time despite persistent infection (34).

This study highlights potential limitations of the OspA vaccine and underscores the notion that quantitatively greater degrees of protection may be conferred by immunization with the molecules responsible for infection-derived immunity. Our view is that recently described outer membrane spanning (Oms) proteins, including porins (33), may prove to be attractive vaccine candidates that might be used alone or in combination with the OspA protein to provide a higher degree of protection against larger inocula and strains of differing OspA serotypes.

\section{Acknowledgments}

We thank Michael J. Miller for his excellent technical assistance. Statistical analysis was performed by Jeffrey A. Gornbein, Dr. PH, UCLA Statistical/Biomathematical Consulting Clinic.

This study was supported by National Institutes of Health (NIH) grant AI-37312, a gift from Smithkline Beecham, and a gift from Dr. Lin Yeiser Coonan to J.N. Miller, NIH grant AI-29733 to M.A. Lovett, and Training Grant 2-T32-AI-07323 to D.M. Foley.

\section{References}

1. Lyme disease surveillance-United States, 1989-1990. MMWR (Morb. Mort. Wkly. Rep.). 1991; 40:417-421.

2. Burgdorfer, W., A.G. Barbour, S.F. Hayes, J.L. Benach, E. Grunwaldt, and J.P. Davis. 1982. Lyme disease-a tickborne spirochetosis? Science (Wash. DC). 216:1317-1319.

3. Steere, A.C. 1989. Lyme disease. N. Engl. J. Med. 321:586-596.

4. Steere, A.C., S.E. Malawista, J.A. Hardin, S. Ruddy, P.W. Askenase, and W.A. Andiman. 1977. Erythema chronicum migrans and Lyme arthritis: the enlarging clinical spectrum. Ann. Intern. Med. 86:685-698.

5. Steere, A.C., R.L. Grodzicki, A.N. Kornblatt, J.E. Craft, A.G. Barbour, W. Burgdorfer, G.P. Schmid, E. Johnson, and S.E. Malawista. 1983. The spirochetal etiology of Lyme disease. N. Engl. J. Med. 308:733-740.

6. Stanek, G., J. Klein, R. Bittner, and D. Glogar. 1990. Isolation of Borrelia burgdorferi from the myocardium of a patient with longstanding cardiomyopathy. N. Engl. J. Med. 322:249-252.

7. Schmidli, J., T. Hunziker, P. Moesli, and U.B. Schaad. 1988. Cultivation of Borrelia burgdorferi from joint fluid 3 months after treatment of facial palsy due to Lyme borreliosis. J. Infect. Dis. 158:905-906.

8. Logigan, E.L., R.F. Kaplan, and A.C. Steere. 1990. Chronic neurologic manifestations of Lyme disease. N. Engl. J. Med. 323:1438-1444.

9. Pachner, A.R., and A.C. Steere. 1985. The triad of neurologic manifestations of Lyme disease: meningitis, cranial neuritis, and radiculoneuritis. Neurology. 35:47-53.

10. Steere, A.C., W.P. Batsford, and M. Weinberg. 1980. Lyme carditis: cardiac abnormalities of Lyme disease. Ann. Intern. Med. 93:8-16.

11. Steere, A.C., R.T. Schoen, and E. Taylor. 1987. The clinical evolution of Lyme arthritis. Ann. Intern. Med. 107:725-731.

12. Ackerman, R., B. Rehse-Kupper, E. Gollmer, and R. Schmidt. 1988. Chronic neurologic manifestations of erythema migrans borreliosis. Ann. NY Acad. Sci. 539:16-23.

13. Pachner, A.R., P. Duray, and A.C. Steere. 1989. Central nervous system manifestations of Lyme disease. Arch. Neurol. 46:790-795.

14. Garcia-Monco, J.C., and J.L. Benach. 1989. The pathogenesis of Lyme disease. Rheum. Dis. Clin. N. Am. 15:711-726.

15. Fikrig, E., S.W. Barthold, F.S. Kantor, and R.A. Flavell. 1990. Protection of mice against the Lyme disease agent by immunizing with recombinant OspA. Science (Wash. DC). 250:553-556.

16. Fikrig, E., S.W. Barthold, F.S. Kantor, and R.A. Flavell. 1992. Long term protection of mice from Lyme disease by vaccination with OspA. Infect. Immun. 60:773-777.

17. Fikrig, E., S.W. Barthold, N. Marcantonio, K. Deponte, F.S. Kantor, and R.A. Flavell. 1992. Roles of OspA, OspB, and flagellin in protective immunity to Lyme borreliosis in laboratory mice. Infect. Immun. 60:657-661.

18. Simon, M., U.E. Schaible, M.D. Kramer, C. Eckerskorn, C. Museteanu, H.K. Muller-Hermelink, and R. Wallich. 1991. Recombinant outer surface protein A from Borrelia burgdorferi induces antibodies protective against spirochetal infection in mice. J. Infect. Dis. 164:123-132.

19. Erdile, L.F., M. Brandt, D.J. Warakomski, G.J. Westrack, A. Sadziene, A.G. Barbour, and J.P. Mays. 1993. Role of attached lipid in immunogenicity of Borrelia burgdorferi OspA. Infect. Immun. 61:81-90.

20. Coughlin, R.T., D. Fish, T.N. Mather, J. Ma, C. Pavia, and P. Bulger. 1995. Protection of dogs from Lyme disease with a vaccine containing outer surface protein A (OspA), OspB, and Saponin adjuvant QS21. J. Infect. Dis. 171: 1049-1052.

21. Fikrig, E., S.R. Telford III, S.W. Barthold, F.S. Kantor, A. Spielman, and R. Flavell. 1992. Elimination of Borrelia burgdorferi from vector ticks feeding on OspA-immunized mice. Proc. Natl. Acad. Sci. USA. 89:5418-5421.

22. Kurtti, T.J., U.G. Munderloh, C.A. Norton Hughes, S.M. Engstrom, and R.C. Johnson. 1996. Resistance to tick-borne spirochete challenge induced by Borrelia burgdorferi strains that differ in expression of outer surface proteins. Infect. Immun. 64:4148-4153.

23. Preac-Mursic, V., B. Wilske, E. Patsouris, S. Jauris, G. Will, E. Soutschek, S. Reinhardt, G. Lehnert, U. Klockmann, and P. Mehraein. 1992. Active immunization with pC protein of Borrelia burgdorferi, protects gerbils against B. burgdorferi infection. Infection. 20:342-349.

24. Probert, W.S., and R.B. LeFebvre. 1994. Protection of C3H/HeN mice from challenge with Borrelia burgdorferi through active immunization with OspA, OspB, or OspC, but not with OspD or the 83-kilodalton antigen. Infect. Immun. 62:1920-1926.

25. Brusca, J.S., A.W. McDowell, M.V. Norgard, and J.D. Radolf. 1991. Localization of outer surface protein A and B in both the outer membrane and in tracellular compartments of Borrelia burgdorferi. J. Bacteriol. 173:8004-8008.

26. Foley, D.M., R.J. Gayek, J.T. Skare, E.A. Wagar, C.I. Champion, D.R. Blanco, M.A. Lovett, and J.N. Miller. 1995. Rabbit model of Lyme borreliosis: erythema migrans, infection-derived immunity, and identification of Borrelia burgdorferi proteins associated with virulence and protective immunity. J. Clin. Invest. 96:965-975.

27. Barbour, A.G. 1984. Isolation and cultivation of Lyme disease spirochetes. Yale J. Biol. Med. 57:521-525.

28. Schwan, T.G., W. Burgdorfer, M.E. Schrumpf, and R.H. Karstens. 1988. The urinary bladder: a consistent source of Borrelia burgdorferi in experimentally infected white-footed mice (Peromyscus leocopus). J. Clin. Microbiol. 26: 893-895.

29. Philipp, M.T., M.K. Aydintug, R.P. Bohm, Jr., F.B. Cogswell, V.A. Dennis, H.N. Lanners, R.C. Lowrie, Jr., E.D. Roberts, M.D. Conway, M. Karacorlu, et al. 1993. Early and early disseminated phases of Lyme disease in the rhesus monkey: model for infection in humans. Infect. Immun. 61:3047-3059.

30. Fikrig, E., S.W. Barthold, D.H. Persing, X. Sun, F.S. Kantor, and R.A. Flavell. 1992. Borrelia burgdorferi strain 25015: characterization of outer surface protein A and vaccination against infection. J. Immunol. 148:2256-2260.

31. Luft, B.J., R.J. Dattwyler, R.C. Johnson, S.W. Luger, E.M. Bosler, D.W. Rahn, E.J. Masters, E. Grunwaldt, and S.D. Gadgil. 1996. Azithromycin compared with amoxicillin in the treatment of erythema migrans. A double-blind, randomized, controlled trial. Ann. Intern. Med. 124:785-791.

32. Johnson, B.J., S.L. Sviat, C.M. Happ, J.J. Dunn, J.C. Frantz, L.W. 
Mayer, and J. Piesman. 1995. Incomplete protection of hamsters vaccinated with unlipidated OspA from Borrelia burgdorferi infection is associated with low levels of antibody to an epitope defined by mAb LA-2. Vaccine. 13:10861094.

33. Skare, J.T., E.S. Shang, D.M. Foley, D.R. Blanco, C.I. Champion, T. Marzabekov, Y. Sokolov, B.L. Kagan, J.N. Miller, and M.A. Lovett. 1995. Virulent strain associated outer membrane proteins of Borrelia burgdorferi. J. Clin. Invest. 96: 2380-2392.

34. Aydintug, M.K., Y. Gu, and M.T. Phillip. 1994. Borrelia burgdorferi antigens that are targeted by antibody-dependent, complement-mediated killing in the rhesus monkey. Infect. Immun. 62:4929-4937.

35. Schaible, U.E., L. Gern, R. Wallich, M.D Kramer, M. Prester, and M.M. Simon. 1993. Distinct patterns of protective antibody are generated against Borrelia burgdorferi in mice experimentally inoculated with high and low doses of antigen. Immunol. Let. 36:219-226.

36. Barthold, S.W., and L.K. Bockenstedt. 1993. Passive immunizing activity of sera from mice infected with Borrelia burgdorferi. Infect. Immun. 61: 4696-4702.

37. Schwan, T.G., J. Piesman, W.T. Golde, M.C. Dolan, and P.A. Rosa.
1995. Induction of an outer surface protein Borrelia burgdorferi during tick feeding. Proc. Natl. Acad. Sci. USA. 92:2909-2913.

38. Barthold S.W., E. Fikrig, L.K. Bockenstedt, and D.H. Persing. 1995. Circumvention of outer surface protein A immunity by host-adapted Borrelia burgdorferi. Infect. Immun. 63:2255-2261.

39. Wilske, B., V. Preac-Mursic, U.B. Gobel, B. Graf, S. Jauris, E. Soutschek, E. Schwab, and G. Zumstein. 1993. An OspA serotyping system for Borrelia burgdorferi based on reactivity with monoclonal antibodies and OspA sequence analysis. J. Clin. Microbiol. 31:340-350.

40. Rosa, P.A., T. Schwan, and D. Hogan. 1992. Recombination between genes encoding major outer surface proteins A and B of Borrelia burgdorferi. 1992. Mol. Microbiol. 6:3031-3040.

41. Jonsson, M., L. Noppa, A.G. Barbour, and S. Bergstrom. 1992. Heterogeneity of outer membrane proteins in Borrelia burgdorferi: comparison of Osp operons of three isolates of different geographic origins. Infect. Immun. 60: 1845-1853.

42. Marconi, R.T., M.E. Konkel, and C.F. Garon. 1993. Variability of Osp genes and gene products among species of Lyme disease spirochetes. Infect. Immun. 61:2611-2617. 